\title{
Waveguide tapers fabrication by femtosecond laser induced element redistribution in glass
}

\author{
Manuel Macias-Montero, Antonio Dias, Belen Sotillo, Pedro Moreno-Zárate, Rocio Ariza, Paloma \\ Fernandez and Javier Solis
}

\begin{abstract}
Here in we present the fabrication and performance of waveguide tapers produced by femtosecond laser induced element redistribution in modified phosphate glasses. More particularly, it is demonstrated that by controlling the scan velocity during the writing process it is possible to adequately tune both the size of the modified area and the refractive index contrast to produce waveguides that can cope with mode field diameters in the range of 7-16 $\mu \mathrm{m}$. In addition, we fabricated tapered structures through the induction of an acceleration in the laser scanning velocity, resulting in a device that can efficiently convert a wide range of mode fields. The fine control achieved over the index contrast in the range of $10^{-3}$ and $10^{-2}$ would allow the production of a wide variety of tapers that could potentially be used to couple numerous photonic devices.
\end{abstract}

Index Terms - fs-laser writing, phosphate glasses, ion migration, optical waveguides, waveguide tapers.

\section{INTRODUCTION}

Femtosecond laser writing is a well stablished procedure to develop photonic devices $[1,2,3,4]$. However, the majority of the structures created by laser writing in glass present a relatively small refractive index contrast [1] $(\Delta \mathrm{n})$ that is bound to the nature of the material itself and to the associated (structural) modification mechanism responsible for the index change [5,6]. Despite these limitations, the use of different writing strategies (e.g. the use of multi-scan writing [7] or depressed cladding structures [8]) has enabled the fabrication of photonic devices in a wide variety of glass families including silicates, phosphates, tellurites, borates, and chalcogenides [1]. Alternatively, the use of fs-laser induced local compositional changes (element redistribution) [9] emerges as a very attractive route to produce refractive microstructures with large index contrast $[2,10]$. Indeed, recent works have shown that femtosecond laser induced element redistribution (FLIER) enables the production of highly efficient waveguides, optical amplifiers and lasers $[2,11,12,13]$ in a highly versatile and controllable manner. The key to

This work was funded through grant numbers TEC2017-82464-R, MAT2017-87035-C2-1-P, and RTI2018-096498-B-I00 (MCIU/AEI/FEDER, EU)."

M. Macias-Montero, A. Dias and J. Solis are with the Laser Processing Group, Instituto de Optica (IO-CSIC), Serrano 121, 28006 Madrid, Spain. (emails: manuel.macias@csic.es; j.solis@io.cfmac.csic.es)

B. Sotillo and P. Fernandez are with Department of Materials Physics, Faculty of Physics, University Complutense of Madrid, Madrid 28040, Spain.

P. Moreno-Zárate is with Electronic Engineering Department, Higher Technological Institute of Acatlan Osorio, Carretera Acatlan - San Juan Ixcaquistla k.m. 5.5, 74949, Acatlan de Osorio, Mexico.

R. Ariza is with the Laser Processing Group, Instituto de Optica (IOCSIC), Serrano 121, 28006 Madrid, Spain and with Department of Materials Physics, Faculty of Physics, University Complutense of Madrid, Madrid 28040, Spain. this technique lies in the addition of small amounts of heavy (such as $\mathrm{La}_{2} \mathrm{O}_{3}, \mathrm{BaO}$ ) and light (typically alkaline) oxide glass modifiers that cross migrate under certain conditions [14] upon fs-laser irradiation, giving rise to $\Delta \mathrm{n}$ values above $10^{-2}[2,10,11,12]$.

Various are the technologies for the production of light guiding devices, including optical fibers, ion-exchanged planar waveguides, silicon on insulator (SOI) waveguides, ... [15]. However, each technology has its own requirements and, in many cases, the produced structures are not directly compatible with one another. For this reason, the development of mode coupling devices started a few decades ago [16]. Even in a single photonic device, as underlined by $\mathrm{Fu}$ et al. [17], waveguides are also likely to be designed with different cross-sections to realize different functions, like arrayed-waveguide gratings [3], multimode interference couplers [18], grating couplers [19], etc. Tapered structures are therefore needed to facilitate the tuning of modal distributions between two light guiding elements at a reduced cost in terms of losses. Waveguide tapers are thus key elements in integrated photonics and play a key role in the development of SOI photonics where small mode field diameters (MFD's) are a must [20,21].

In terms of design, several works have shown that complex mathematically designed structures represent an improvement compared to more classical approaches such as linear or parabolic assemblies $[22,23]$. In terms of fabrication, the increasing number of components in photonic chips makes the simultaneous reduction of the physical size and inherent losses of tapers to be challenging. In semiconductors and SOI devices, most fabrication procedures involve UV photolithography-based techniques, keeping the material/s index properties as fixed parameters [24]. Alternatively, fabrication of optimized tapers can be achieved through a controlled modification of the index contrast created into one or more local regions by a combination of different materials [25].

This later approach (local index control) has been successfully combined with the inherent versatility of femtosecond laser direct write for the production of ultra-broadband integrated tapered mode multiplexers [26], where the local control of the index of the structures was achieved by ramping the power of the writing laser acting on a borosilicate glass (Corning Eagle 2000). Similarly, arrayed waveguide gratings (AWG's) with taper arrays have been more recently demonstrated by fs-laser writing in the same glass [3] using a modest index contrast $\left(\Delta \mathrm{n} \sim 1.5 \times 10^{-3}\right)$. The increasing interest of quantum computing has also led to the production of tapered fslaser written waveguides in fused silica for fiber in-coupling and outcoupling of the waveguide structures where the quantum operations are performed [27]. In fact, there are commercial products on the market that employ femtosecond laser written tapers in their design. Yet, it is worth noting though that conventional fs-laser writing in glass is hampered by the index contrast limitations above indicated. Alternatively, although very reproducible, the conditions to create high index contrast compositional structures by FLIER are up to some extent limited by width of the processing windows where smooth, and stable ion migration processes can be induced upon laser 
exposure in a moving sample.

Here in, we propose a new approach to overcome such limitation to produce tunable waveguides in terms of the size of the guiding region and its index contrast. The advances attained have enabled us to fabricate optical tapers that effectively transform a wide range of mode field diameters with low propagation losses in modified phosphate glasses. The developed approach can be similarly used in other glass families and shows a large potential for rapid prototyping of efficient integrated photonic components involving taper structures. The emphasis of this work lays on the use of a novel approach, like FLIER, to generate light guiding structures where local index can be finely tuned.

\section{DEVICE FABRicATION AND CHARACTERIZATION}

Waveguides are fabricated in compositionally customized glass $\left(67 \mathrm{P}_{2} \mathrm{O}_{5}-7 \mathrm{Al}_{2} \mathrm{O}_{3}-6 \mathrm{SiO}_{2}-8 \mathrm{~K}_{2} \mathrm{O}-8 \mathrm{La}_{2} \mathrm{O}_{3}-1 \mathrm{Er}_{2} \mathrm{O}_{3}-2 \mathrm{Yb}_{2} \mathrm{O}_{3}\right.$ mol.\%) made by Glass Technology Services Ltd. A high-repetition-rate fiberbased femtosecond laser beam is used to perform the inscription (Satsuma Amplitude Systems). A schematic representation of the experimental setup is presented in Figure 1. As shown in this figure, fs pulses (350 fs) at $1030 \mathrm{~nm}$ are put through a quarter-wave plate $(\lambda / 4)$, a $1.3 \mathrm{~mm}$ slit and an aspheric lens of 0.68 numerical aperture that focuses the beam $150 \mu \mathrm{m}$ underneath the surface, while scanning $12 \mathrm{~mm}$ long samples at velocities ranging from 60 to $900 \mu \mathrm{m} / \mathrm{s}$. For the production of the tapers, irradiations where performed using an initial velocity of $60 \mu \mathrm{m} / \mathrm{s}$ for the first $2 \mathrm{~mm}$ and then samples were accelerated for the next $10 \mathrm{~mm}$ to reach the desired final velocity $(100-900 \mu \mathrm{m} / \mathrm{s})$ at the rear of the sample. This process is carried out at constant pulse energy. For each experimental condition, at least three devices are produced.

Optical cross section and longitudinal images are taken in a Nikon Eclipse optical microscope with a $100 \times, 0.9$ NA objective lens. Near field guided modes are analyzed by coupling a SMF-28 single mode fiber and imaging with a 50× objective and an infrared camera (Goodrich SUI) at $976 \mathrm{~nm}$ and 1440-1640 $\mathrm{nm}[11,13]$. Alternatively, taper structures are also analyzed coupling a M67L01 25 $\mu \mathrm{m} 0.10 \mathrm{NA}$ fiber. Overall losses evaluation is performed by coupling either type of optical fiber and measuring the output power of each waveguide with a photodiode sensor (Ophir PD300) at $1640 \mathrm{~nm}$ to be sufficiently far from $\mathrm{Er}^{3+}$ absorption band. Propagation losses are obtained after subtraction of coupling and Fresnel losses, subsequently dividing by the structure length. Local elemental composition is obtained using energy-dispersive X-ray microanalysis (Bruker AXS Quantax $\mu$-analysis) in a scanning electron microscope (Leica S440).

\section{EXPERIMENTAL RESULTS AND DISCUSSION}

High repetition rate, femtosecond-laser writing in $\mathrm{La}_{2} \mathrm{O}_{3} \cdot \mathrm{K}_{2} \mathrm{O}$ modified phosphate glass induces local refractive index changes by element redistribution above a certain energy threshold [14]. However, well performing waveguides are only formed within a relatively narrow pulse energy window of $\sim 500-600 \mathrm{~nJ}$ for the present composition when scanning at $60 \mu \mathrm{m} / \mathrm{s}$. Outside this interval, energy coupling instabilities lead to spatial discontinuities in the FLIER process that dramatically increase propagation losses. As a consequence, although tuning the index contrast of the waveguides is feasible [12], a robust, fine tuning of $\Delta \mathrm{n}$ via pulse energy alone is challenging due to the constrains imposed by the width of the stability region.

To avoid such constrains while tuning the index, we have explored the possibility of performing laser treatments with different sample scanning velocities, ranging from 60 to $900 \mu \mathrm{m} / \mathrm{s}$. Optical microscopy cross section images of the produced light guiding structures for increasing writing scanning speeds are included in Figure 2. In these images, it is possible to observe that the bright area, that corresponds to a local refractive index increase [9,11], decreases in size with the sample scanning speed. In all cases, the bright part is located above the darker area, sharing a well define border between them. This ensures good guiding properties, as it is noted later in the present paper. To quantify the size reduction, measurements were carried out using optical microscopy images for a variety of scanning velocities and two irradiation energies, 560 and $590 \mathrm{~nJ}$. A representation of the data retrieved from these measurements is shown in Figure 2d. This graph shows that the guiding area decreases from 35 to $12 \mu \mathrm{m}^{2}$, almost a factor of 3 , by increasing the scanning velocity from 60 to $900 \mu \mathrm{m} / \mathrm{s}$. Furthermore, both writing energies seem to follow a similar tendency, which is in agreement with the apparent independence between size and irradiation energy in the energy stability window for a given scanning speed, something already discussed in [12].

Additionally, morphological and compositional analysis of samples has been performed by means of scanning electron microscopy (SEM) and energy dispersive X-ray microanalysis (EDX). In agreement with previous studies [11,12], EDX line-scan and mapping included in Figure $3 \mathrm{a}$ )-b) show that the brighter area observed in the cross-section corresponds to a local enrichment of $\mathrm{La}_{2} \mathrm{O}_{3}$ and a depletion of $\mathrm{K}_{2} \mathrm{O}$, while the opposite occurs in the darker part. Analyzing the relative enrichments of both $\mathrm{K}$ and $\mathrm{La}$ for the different scanning velocities, it is possible to observe that the element redistribution in the modified regions becomes weaker for higher velocities. As it can be seen in Figure $3 \mathrm{c}$ ), in the case of La the enrichment decreases from 20 to $10 \%$, and for $\mathrm{K}$ the depletion reduces from 48 to $30 \%$. All the other chemical components of the glass were studied conjointly, although no significant modifications were observed in the EDX data in agreement with [11-13]. Therefore, the consequence of increasing the sample scanning velocity during writing is twofold, the modified area decreases in size and the $\mathrm{La}$ enrichment reduces. The modification of the steady state heat flow and diffusion processes in the modifying region for increasing sample speeds accounts for this effect.

To evaluate the performance of the waveguides written at different sample speeds, the mode fields propagated for different wavelengths were put through. Figures 4 a) and b) show the results for $1640 \mathrm{~nm}$ input wavelength. There, it is possible to observe that the MFD increases in parallel with the scanning velocity, varying between 7 to $16 \mu \mathrm{m}$. The increment in size is particularly rapid in the case of 590 $\mathrm{nJ}$ writing pulse energy, reaching a saturation limit at around $16 \mu \mathrm{m}$. This behavior is consistent with previous studies on the effect of scanning speed on the MFD [28]. The fact that the MFD's of waveguides produced at $590 \mathrm{~nJ}$ are greater than those of $560 \mathrm{~nJ}$ is related to a poorer light confinement due to the closeness of this condition to the edge of the energy stability window (upper limit, 600 $\mathrm{nJ})$ that leads to structures with less sharpen edges and a lower concentration of heavy modifiers in the guiding region. This is further confirmed by refractive index contrast $(\Delta \mathrm{n})$ estimations. To obtain $\Delta \mathrm{n}$, we use Marcuse's experimental equation that relates the size of the guiding area, the MFD and the normalized frequency (V number) assuming step-index single-mode waveguides [29]. In photonics, and more particularly for step-index optical fibers, the normalized frequency is a parameter that enables to determine the number of supported modes. This $\mathrm{V}$ number is proportional to the optical frequency, the core radius and the numerical aperture of the guiding structure [30]. We can see in the figure that for $590 \mathrm{~nJ}$, the 
maximum $\Delta \mathrm{n}$ value is reached at the lowest writing speed. The maximum value obtained for $\Delta \mathrm{n}$ was in the order of $1.1 \cdot 10^{-2}$ for 560 $\mathrm{nJ}$ pulses, being possible to controllably lower it down to $5 \cdot 10^{-3}$ at $590 \mathrm{~nJ}$ of energy. The observed trend in $\Delta \mathrm{n}$ and MFD's with respect to scanning velocity can be related to two combined factors. On one hand, increasing the velocity results in a decrease in the number of pulses per unit area, therefore reducing both the energy delivered and the modified area. On the other hand, the ion migration process is also affected, lower effective energies lead to a poorer La-enrichment in the guiding area, which results in the lower $\Delta \mathrm{n}$ observed for the highest scanning velocities for both irradiation energies studied [12]. Thus, the scanning velocity is a very convenient experimental parameter that enables to precisely tune the refractive index within glass samples. An extrapolation of the results in Fig. 4 c) indicates that through the control of the pulse energy in the 500-600 $\mathrm{nJ}$ range and of the scanning speed in $100-2000 \mu \mathrm{m} / \mathrm{s}$ it would be feasible to finely tune the index contrast of the structures between $10^{-3}$ and $10^{-2}$. Regarding light propagation, measurements show low losses for the slower velocities $(\sim 1 \mathrm{~dB} / \mathrm{cm})$, while higher ones tend to increase propagation losses.

These results show that the experimental approach used allows to create a range of waveguides with selective $\Delta \mathrm{n}$ that can cope with different MFDs. In addition, the production of these structures is not limited to a single scanning velocity. Indeed, it is possible to accelerate or decelerate the writing process, enabling the transformation of the mode field along the waveguides adiabatically $[16,18]$. Along this direction, experiments were carried out using a scanning velocity of $60 \mu \mathrm{m} / \mathrm{s}$ at the beginning and up to $900 \mu \mathrm{m} / \mathrm{s}$ at the end of the writing process to produce taper structures as presented in Figure 5 a). Results show that, while waveguides produced at various constant scanning velocities fail to compress a large MFD (21.5 $\mu \mathrm{m})$, tapered waveguides written with deceleration do effectively compress such MFD, reducing it to $8 \mu \mathrm{m}$ or less. Figure 5 b) shows the output MFD of both constant velocity waveguides and accelerated ones. In the first case, variation of the speed enables to have waveguides with adaptive MFD's $(8-16 \mu \mathrm{m})$, which renders in a reduction of the coupling losses with respect to the $21.5 \mu \mathrm{m}$ input MFD. However, their lower $\Delta \mathrm{n}$ lead to higher propagation loses, reaching values close to $6 \mathrm{~dB} / \mathrm{cm}$. On the other hand, tapered waveguides can cope with large input MFD's and effectively transform them into smaller ones, of about $8 \mu \mathrm{m}$, in all the cases as it is shown in Figure $5 \mathrm{~b}$ ). Additionally, the structure presents the low coupling losses of large MFD waveguides and smaller propagation losses related to the $\Delta \mathrm{n}$ variation, which results in $1.8 \mathrm{~dB} / \mathrm{cm}$ for acceleration up to $200 \mu \mathrm{m} / \mathrm{s}, 2.4 \mu \mathrm{dB} / \mathrm{cm}$ for $350 \mu \mathrm{m} / \mathrm{s}$ and approximately $4 \mathrm{~dB} / \mathrm{cm}$ for final velocities equal or greater than 500 $\mu \mathrm{m} / \mathrm{s}$. Reversibility tests are also satisfactory, as the tapered structures are able to transform an input MFD of $8 \mu \mathrm{m}$ into a range that goes from 9 to $16 \mu \mathrm{m}$ depending on the final scanning velocity, as indicated by the yellow arrows in Fig. 5, matching the respective results obtained for constant speed waveguides.

To conclude this section, there are some aspects that should be considered regarding the potential of FLIER for the production of efficient taper structures. In the present results, it has to be emphasized that although the size of the guiding regions produced with an index contrast of $\Delta \mathrm{n} \sim 10^{-2}$ is around $6 \mu \mathrm{m}$ in diameter, smaller or larger structures with similarly high $\Delta \mathrm{n}$ could be produced by tuning the numerical aperture of the writing optics and the slit width value used during the writing process [12]. Additionally, we have recently shown [31] that a careful selection of the relative content of moving ions enables maximizing the index contrast achievable in a given glass matrix. These features can strongly broaden the range of
MFD's achievable with FLIER waveguides, from very small to very large values. Along with large multi-mode coupling from and to fibers, coupling and decoupling operations from photonic chips, very specially for the case of quantum operations [32] in silica-based, fslaser written integrated devices $[27,33,34]$ would be thus enabled with low losses. It also has to be emphasized that the index contrast associated to the FLIER structures is spectrally flat at least in the $550-1600 \mathrm{~nm}$ spectral range [35]. This feature is particularly relevant for the production of improved laser written VIS-IR operating AWG's [3] and photonic lanterns for which additionally the large index contrast accessible can help in reducing the bending losses and cross talk in the waveguides array. Finally, refractive functional structures generated by FLIER have been produced generating the cross migration of different heavy and light glass modifiers in several glass families, including phosphates [12], borates [13], tellurites [36] and more recently silicates [37]. In later ones, index contrast values above $2 \times 10^{-2}$ have been observed at IR wavelengths in waveguides produced by FLIER, which indicates that compositional design provides an additional optimization parameter for the production of high performance, versatile taper structures using this technique.

\section{CONCLUSION}

In summary, we have demonstrated that FLIER enables the production of efficient photonic devices in modified phosphate glasses. In particular, the control over the scan velocity offers the possibility of precisely tuning the properties of waveguides, such as the MFD or the index contrast. Moreover, this technology has allowed the fabrication of photonic devices with spatially variable size and index contrast that are capable of effectively transform MFD of up to $21 \mu \mathrm{m}$ to less than $8 \mu \mathrm{m}$. Therefore, these tapered structures are capable of facilitating the transition between different light guiding elements with overall low losses. We foresee that these structures will find practical applications in the interconnection of components such as optical fibers, ion-exchanged planar waveguides or SOI waveguides.

\section{ACKNOWLEDGMENT}

M.M. acknowledges the postdoctoral Juan de la Cierva Incorporación grant (IJCI-2017-33317)) of the Spanish Ministry of Research and Innovation. B.S. acknowledges the postdoctoral grant from the "Atracción de Talento" Program funded by the Comunidad de Madrid (2017-T2/IND-5465). P.M.-Z. acknowledges the postdoctoral scholarship \#235346 awarded by the National Council of Science and Technology from Mexico (CONACYT).

\section{REFERENCES}

[1] R. Osellame, G. Cerullo, and R. Ramponi, "Femtosecond Laser Micromachining: Photonic and Microfluidic Devices in Transparent Materials," Top. Appl. Phys., vol. 123, 2012, doi: 10.1007/978-3-642-23366-1.

[2] A. Dias et al., "Femtosecond laser writing of photonic devices in borate glasses compositionally designed to be laser writable," Opt. Lett., vol. 43, no. 11, p. 2523, Jun. 2018, doi: 10.1364/ol.43.002523.

[3] G. Douglass, F. Dreisow, S. Gross, and M. J. Withford, "Femtosecond laser written arrayed waveguide gratings with integrated photonic lanterns," Opt. Express, vol. 26, no. 2, p. 1497, Jan. 2018, doi: 10.1364/oe.26.001497.

[4] A. Ródenas et al., "Three-dimensional femtosecond laser nanolithography of crystals," Nature Photonics, vol. 13, no. 
2. Nature Publishing Group, pp. 105-109, Feb. 01, 2019, doi: 10.1038/s41566-018-0327-9.

[5] W. J. Reichman et al., "A spectroscopic comparison of femtosecond-laser-modified fused silica using kilohertz and megahertz laser systems," J. Appl. Phys., vol. 99, no. 12, p. 123112, Jun. 2006, doi: 10.1063/1.2207556.

[6] J. D. Musgraves, K. Richardson, and H. Jain, "Laserinduced structural modification, its mechanisms, and applications in glassy optical materials," Opt. Mater. Express, vol. 1, no. 5, p. 921, Sep. 2011, doi: 10.1364/ome.1.000921.

[7] D. G. MacLachlan et al., "Development of integrated mode reformatting components for diffraction-limited spectroscopy," Opt. Lett., vol. 41, no. 1, p. 76, Jan. 2016, doi: 10.1364/ol.41.000076.

[8] S. Gross, M. Ams, D. G. Lancaster, T. M. Monro, A. Fuerbach, and M. J. Withford, "Femtosecond direct-write überstructure waveguide Bragg gratings in ZBLAN," Opt. Lett., vol. 37, no. 19, p. 3999, Oct. 2012, doi: 10.1364/ol.37.003999.

[9] Y. Liu et al., "Micromodification of element distribution in glass using femtosecond laser irradiation," Opt. Lett., vol. 34, no. 2, p. 136, Jan. 2009, doi: 10.1364/ol.34.000136.

[10] T. T. Fernandez et al., "Bespoke photonic devices using ultrafast laser driven ion migration in glasses," Progress in Materials Science, vol. 94. Elsevier Ltd, pp. 68-113, May 01, 2018, doi: 10.1016/j.pmatsci.2017.12.002.

[11] T. Toney Fernandez et al., "Ion migration assisted inscription of high refractive index contrast waveguides by femtosecond laser pulses in phosphate glass," Opt. Lett., vol. 38 , no. 24, p. 5248, Dec. 2013, doi: 10.1364/ol.38.005248.

[12] J. Del Hoyo et al., "Control of waveguide properties by tuning femtosecond laser induced compositional changes," Appl. Phys. Lett., vol. 105, no. 13, p. 131101, Sep. 2014, doi: 10.1063/1.4896846.

[13] G. Escalante, J. Solís, J. del Hoyo, J. A. Vallés, P. Fernández, and P. Moreno-Zárate, "High-Efficiency Waveguide Optical Amplifiers and Lasers via FS-Laser Induced Local Modification of the Glass Composition," $J$. Light. Technol. Vol. 35, Issue 14, pp. 2955-2959, vol. 35, no. 14, pp. 2955-2959, Jul. 2017.

[14] T. T. Fernandez et al., "Dual regimes of ion migration in high repetition rate femtosecond laser inscribed waveguides," IEEE Photonics Technol. Lett., vol. 27, no. 10, pp. 1068-1071, May 2015, doi: 10.1109/LPT.2015.2407378.

[15] G. C. Righini and A. Chiappini, "Glass optical waveguides: a review of fabrication techniques," Opt. Eng., vol. 53, no. 7, p. 071819, Mar. 2014, doi: 10.1117/1.oe.53.7.071819.

[16] A. F. Milton and W. K. Burns, "Mode Coupling in Optical Waveguide Horns," IEEE J. Quantum Electron., vol. 13, no. 10, pp. 828-835, 1977, doi: 10.1109/JQE.1977.1069240.

[17] Y. Fu, T. Ye, W. Tang, and T. Chu, "Efficient adiabatic silicon-on-insulator waveguide taper," Photonics Res., vol. 2, no. 3, p. A41, Jun. 2014, doi: 10.1364/prj.2.000a41.

[18] L. B. Soldano and E. C. M. Pennings, "Optical Multi-Mode Interference Devices Based on Self-Imaging: Principles and Applications," J. Light. Technol., vol. 13, no. 4, pp. 615627, 1995, doi: 10.1109/50.372474

[19] R. Marchetti et al., "High-efficiency grating-couplers: Demonstration of a new design strategy," Sci. Rep., vol. 7, no. 1 , pp. 1-8, Dec. 2017, doi: 10.1038/s41598-017-16505$\mathrm{Z}$.

[20] R. Halir et al., "Waveguide sub-wavelength structures: a review of principles and applications," Laser Photon. Rev., vol. 9, no. 1, pp. 25-49, Jan. 2015, doi: 10.1002/lpor.201400083.

[21] A. Politi, M. J. Cryan, J. G. Rarity, S. Yu, and J. L. O’Brien, "Silica-on-silicon waveguide quantum circuits," Science (80-. )., vol. 320, no. 5876, pp. 646-649, May 2008, doi: $10.1126 /$ science. 1155441 .

[22] B. Luyssaert, P. Bienstman, P. Vandersteegen, P. Dumon, and R. Baets, "Efficient Nonadiabatic Planar Waveguide Tapers," J. Light. Technol. Vol. 23, Issue 8, pp. 2462-, vol. 23, no. 8, p. 2462, Aug. 2005.

[23] H.-T. Chen and K. J. Webb, "Silicon-on-insulator irregular waveguide mode converters," Opt. Lett., vol. 31, no. 14, p. 2145, Jul. 2006, doi: 10.1364/ol.31.002145.

[24] H. Zhou, J. Song, C. Li, H. Zhang, and P. G. Lo, “A library of ultra-compact multimode interference optical couplers on SOI," IEEE Photonics Technol. Lett., vol. 25, no. 12, pp. 1149-1152, 2013, doi: 10.1109/LPT.2013.2261193.

[25] M. Papes et al., "Fiber-chip edge coupler with large mode size for silicon photonic wire waveguides," Opt. Express, vol. 24, no. 5, p. 5026, Mar. 2016, doi: 10.1364/oe.24.005026.

[26] S. Gross, N. Riesen, J. D. Love, and M. J. Withford, "Threedimensional ultra-broadband integrated tapered mode multiplexers," Laser Photon. Rev., vol. 8, no. 5, pp. L81L85, Sep. 2014, doi: 10.1002/lpor.201400078.

[27] R. Heilmann, C. Greganti, M. Gräfe, S. Nolte, P. Walther, and A. Szameit, "Tapering of femtosecond laser-written waveguides," Appl. Opt., vol. 57, no. 3, p. 377, Jan. 2018, doi: 10.1364/ao.57.000377.

[28] T. Meany et al., "Towards low-loss lightwave circuits for non-classical optics at 800 and 1,550 nm," Appl. Phys. A vol. 114, no. 1, pp. 113-118, 2014, doi: 10.1007/s00339013-8090-8.

[29] C. D. Hussey and F. Martinez, "Approximate Analytic Forms For The Propagation Characteristics Of Single-Mode Optical Fibres," Electron. Lett., vol. 21, no. 23, pp. 11031104, 1985, doi: 10.1049/el:19850783.

[30] A. W. Snyder and J. D. Love, Optical Waveguide Theory. London, UK: Chapman and Hall, 1983.

[31] P. Moreno-Zarate et al., "Role of the $\mathrm{La} / \mathrm{K}$ Compositional Ratio in the Properties of Waveguides Written by Fs-Laser Induced Element Redistribution in Phosphate-Based Glasses," Materials (Basel)., vol. 13, no. 6, p. 1275, Mar. 2020, doi: 10.3390/ma13061275.

[32] E. Knill, R. Laflamme, and G. J. Milburn, "A scheme for efficient quantum computation with linear optics," Nature, vol. 409, no. 6816, pp. 46-52, Jan. 2001, doi: $10.1038 / 35051009$.

[33] S. Weimann et al., "Implementation of quantum and classical discrete fractional Fourier transforms," Nat. Commun., vol. 7, no. 1, pp. 1-8, Mar. 2016, doi: 10.1038/ncomms11027.

[34] A. Crespi et al., "Suppression law of quantum states in a 3D photonic fast Fourier transform chip," Nat. Commun., vol. 7, no. 1, pp. 1-8, Feb. 2016, doi: 10.1038/ncomms 10469.

[35] P. Moreno-Zarate et al., "Imaging Ellipsometry Determination of the Refractive Index Contrast and Dispersion of Channel Waveguides Inscribed by fs-Laser Induced Ion-Migration," Phys. status solidi, vol. 215, no. 19, p. 1800258, Oct. 2018, doi: 10.1002/pssa.201800258.

[36] T. T. Fernandez et al., "Role of ion migrations in ultrafast laser written tellurite glass waveguides.," Opt. Express, vol. 22, no. 12, pp. 15298-304, Jun. 2014, doi: 10.1364/OE.22.015298.

[37] M. Macias-Montero et al. "Element redistribution assisted femtosecond laser writing of waveguides in glasses: from 
mechanisms to devices," presented at the OSA Advanced Photonics Congress, Montreal, Canada, Jul. 13-16, 2020. 